\title{
Material design of a biomimetic composite material used for a wooden building joint structure
}

\author{
T. Ota ${ }^{1} \&$ S. Enoki ${ }^{2}$ \\ ${ }^{I}$ Advanced Engineering Student, \\ Nara National College of Technology, Japan \\ ${ }^{2}$ Department of Mechanical Engineering, \\ Nara National College of Technology, Japan
}

\begin{abstract}
Earthquake-resistant wooden houses have been built in Japan for a long time. It has been clear that the buildings absorb the vibration energy in the joint parts of the wooden structures. The mechanism of energy absorption distorts the wood of the joint. Therefore, it is important to develop joint parts that can be easily exchanged for continued absorption of such energy. It has been reported that biomimetic composite materials that imitate bone have a load decentralization function and a shock-absorbing function. Wood has cell structures that resemble bone. Therefore, it seems a logical step to consider biomimetic composite materials that can be applied to wooden buildings. In this study, we propose to create a wooden building joint using a biomimetic composite material. The joint is constructed of aluminum lattice and plastic. In order to test whether this joint structure would successfully absorb energy from earthquake vibrations, in this paper we present results from an investigation into material characteristics. Linear analyses were executed on the material. Load-displacement curves were made by the constraint displacements and calculated reaction forces. Results indicated that the material has nonlinear characteristics. We infer that the composite aluminum and plastic material absorbs the vibration energy because of its nonlinear characteristics. Based on study results, we conclude that using this composite material for proposed joints can have significant benefits towards the earthquake-proofing of individual houses.
\end{abstract}

Keywords: material design, biomimetic composite material, wooden structures, joints and structural nonlinearity. 


\section{Introduction}

There has been a growing need for safety of buildings in recent years. Therefore, great earthquake-proof buildings have been developing. By using the latest technology, e.g. seismic isolation rubber, it has become possible to absorb quakes and to ensure safety even on skyscraper buildings. Japan is prone to earthquakes. Houses that adjust to the earthquakes have been built in Japan for a long time. The Japanese houses are made of wood. It was clarified that the wooden buildings absorbed and emanated the vibration energy and the shock energy in the joint part of the buildings. The mechanism of energy absorption is that wood dents in the joint part $[1,2]$. It is likely that the joint part will be damaged after absorbing the energy once. The houses, as important Japanese cultural properties, are repaired at enormous cost. However it is not possible to spend a large amount of money on conventional wooden buildings. Therefore, it is important to develop joint parts that can be easily exchanged for the continued absorption of such energy.

It has been reported that biomimetic composite materials that imitate bone have a load decentralization function and a shock-absorbing function [3]. Wood has cell structures that resemble bone. Therefore, it seems a logical step to consider biomimetic composite materials that can be applied to wooden buildings.

In this study, we propose wooden building joints using biomimetic composite materials and discuss the material design. Existing quake-absorbing joints are bolted to columns and there is concern that the bolts reduce the strength of the columns. The proposed joints have no bolts and are just put between columns.

\section{Composite materials}

The reported biomimetic composite material, which has a load decentralization function and a shock-absorbing function, is a hexagonal honeycomb structure and a solid-fluid composite. We propose composite materials based on the

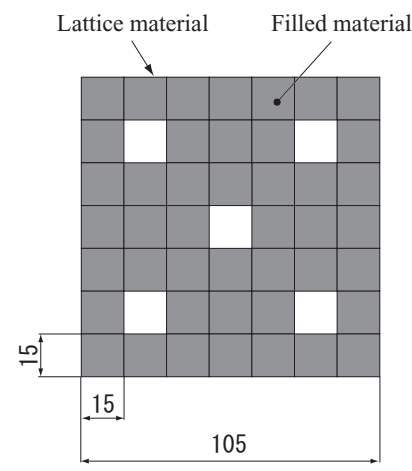

Figure 1: Composite material.

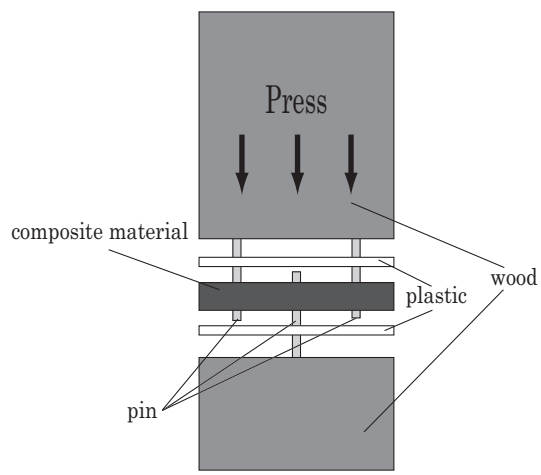

Figure 2: Assembly. 
honeycomb solid-fluid composite. The proposed composite materials consist of a lattice structure with $15 \mathrm{~mm} \times 15 \mathrm{~mm} \times 15 \mathrm{~mm}$ square cells (thickness $0.5 \mathrm{~mm}$ ) and a filled material, as shown Fig.1. The square lattice structure is made of high rigidity thin-material and is filled up with low rigidity material in the cells as shown in the gray parts in Fig.1. Five pins are put in four corners cells and a center cell as shown in the white parts in Fig.1. The pins connect a groundsill and a column with a proposed composite material. Plastic plates are put on as shown in Fig. 2 in order to avoid contact of the wooden groundsill and the column with the composite material. The thickness of the plates is $1 \mathrm{~mm}$.

\section{Material design}

\subsection{Proposed design method}

In this study, we focus a nonlinear characteristic in the elastic region. Rubbers have a load decentralization function and a shock absorbing function because of the nonlinear characteristics. We examine the composite material characterization. Generically, the elastic region has a linear relation in Loaddisplacement curves. We guess that the composite materials have structural nonlinearity in the elastic region. We checked the results, calculating by 3D linear analyses whether the load-displacement curves of the composite materials indicate nonlinearity. We can carry out material design by using the analyses if the results meet the requirements.

\subsection{Analysis method and condition}

General-purpose FEM software "ANSYS" is used on the analyses in this study. Elements used in this study are 4 nodes shell elements on the square lattice structure and 8 nodes solid elements on filled material in the cells. The analyses assume that a quake occurs. Equivalent constraint displacements of some quake energies apply a composite material from a center pin. The energies transmit from the center pin to other pins in four corners. Reaction forces occur on the faces in contact with the four corners pins.

The boundary conditions are showed in Fig.3. Constraint displacements are set up on the faces in contact with the center pin. The displacements are 0.001$1 \mathrm{~mm}$. The faces in contact with the four corner pins are restrained. The directions of the constraint displacements are $90 \mathrm{deg}$. and $45 \mathrm{deg}$., as shown in Fig. 3. In the case of $45 \mathrm{deg}$., constraint displacements are set up such that the contact faces shift parallel, as shown in Fig.4.

The material properties are showed in Table 1. In this paper, we calculate two kinds of composite materials. One of them consists of an aluminum lattice and a filled plastic. The other consists of an aluminum lattice and a filled imaginary material that have quite low longitudinal elastic modulus. We also calculate a joint formed by a plastic alone. 


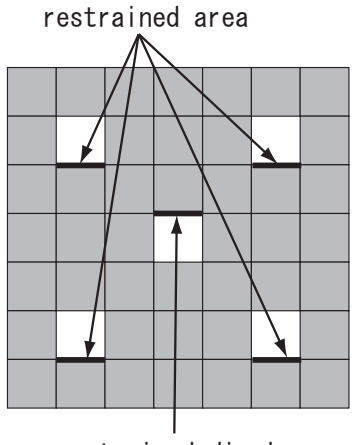

constrained displacement

(a) $90 \mathrm{deg}$.

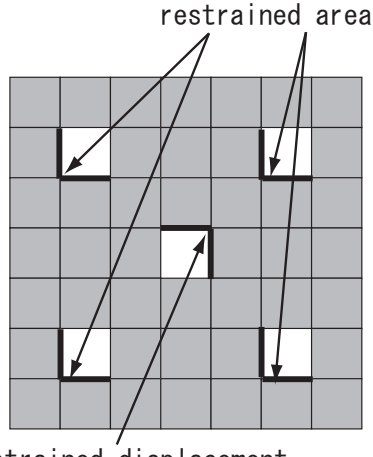

constrained displacement

(b) 45 deg.

Figure 3: Boundary conditions.

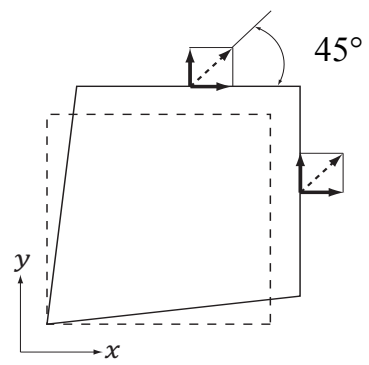

Figure 4: Constraint displacement (45 deg.).

Table 1: $\quad$ Material properties.

\begin{tabular}{|c|c|c|c|}
\hline & Material & $\begin{array}{c}\text { Longitudinal } \\
\text { modulus } \\
{[\mathrm{MPa}]}\end{array}$ & $\begin{array}{c}\text { Poisson ratio } \\
{[-]}\end{array}$ \\
\hline $\begin{array}{c}\text { High rigidity } \\
\text { thin material }\end{array}$ & Aluminum & 70000 & 0.33 \\
\hline \hline $\begin{array}{c}\text { Low rigidity } \\
\text { material }\end{array}$ & $\begin{array}{c}\text { Imastic } \\
\text { material }\end{array}$ & 1000 & 0.3 \\
\cline { 2 - 4 } & \multicolumn{2}{|c}{} \\
\hline
\end{tabular}

\section{Analysis results and consideration}

\subsection{Characteristics of the composite materials}

Load-displacement curves are charted by using some constraint displacements and total reaction forces of all restrained points. We evaluate material characterizations of the materials in Table 1 from the curves. 
Firstly, displacement distributions and load-displacement curves in the case of the 90 deg. direction are shown in Figs.5 and 6. It is understood that there is no difference between the displacement distributions of all materials and that the distribution of the deformation is not even, as shown in Fig.5. Relations between reaction forces and displacements of all materials are not nonlinear in this direction case, as shown in Fig.6.

The displacement distributions and load-displacement curves in the case of the 45 deg. direction are shown in Figs.7 and 8. Each displacement distribution of the composite materials is more symmetrical in a transverse and a longitudinal direction than that of the plastic material, as shown in Fig.7. Therefore, it is understood to have distributed the deformation evenly when the composite materials with the square lattice received the load in the direction of $45 \mathrm{deg}$. Fig. 8 indicates that reaction forces of the composite materials increase rapidly when the constraint displacements act and it is thought that the reaction forces decrease until the constraint displacements become $0.01 \mathrm{~mm}$. The results indicate that the composite materials have structural nonlinearity within a range of small displacements. On the other hand, there is a bias on the displacement

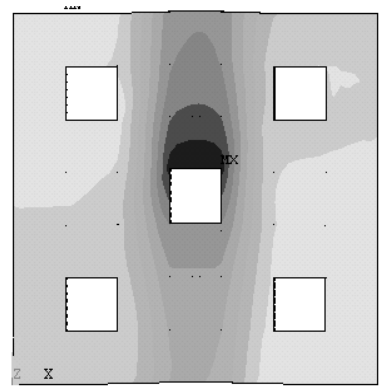

(a) Aluminum-plastic

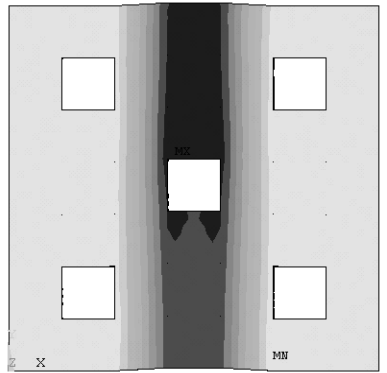

(b) Aluminum-imaginary material

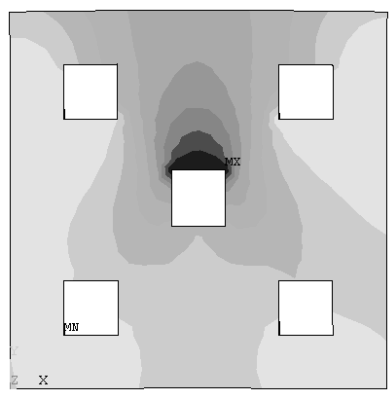

(c) Plastic

Figure 5: Displacement distribution (90 deg.). 
334 Design and Nature V

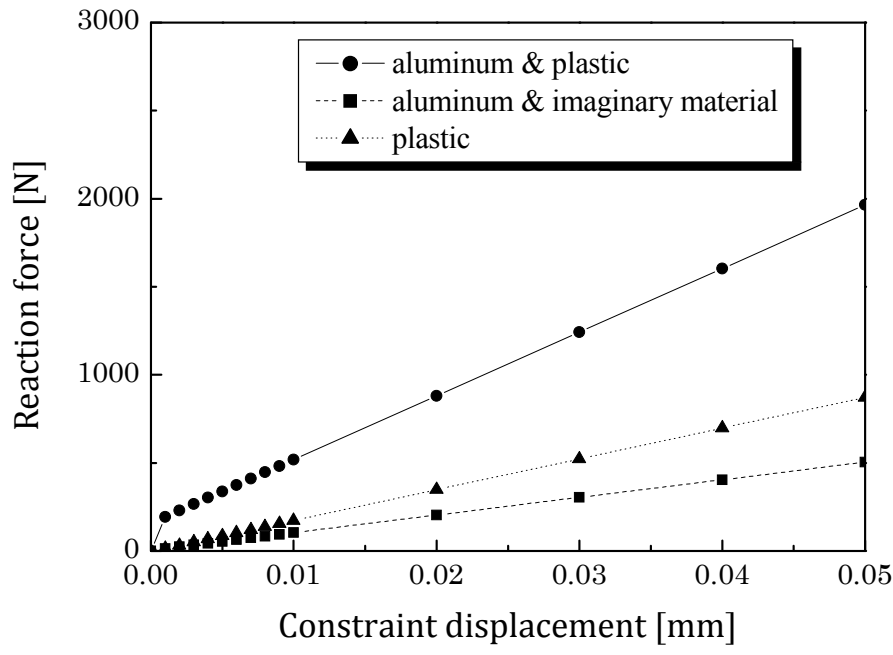

Figure 6: Load-displacement curves (90 deg.).

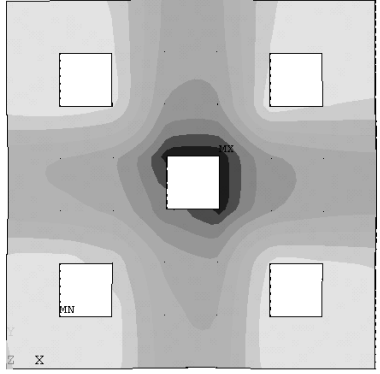

(a) Aluminum-plastic

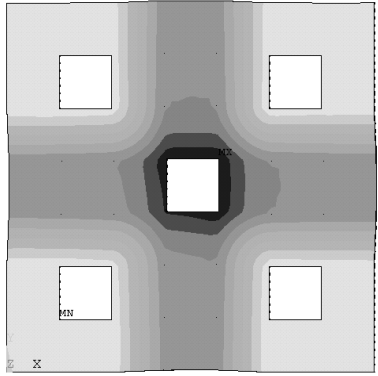

(b) Aluminum-imaginary material

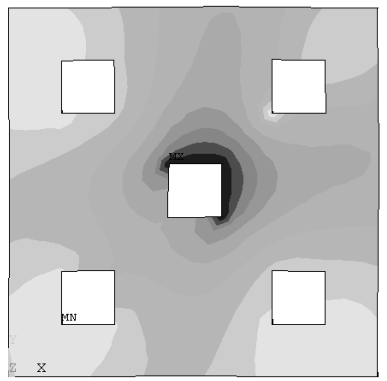

(c) Plastic

Figure 7: Displacement distribution (45 deg.). 
distribution of the plastic and the energy concentrates in the direction of the constraint displacement, as shown in Fig.7(c). The reaction force of the plastic increases as well as the composite materials after the constraint displacement act; however, the relation between the displacement and the reaction force becomes linear, as shown in Fig.8. This result does not indicate that the plastic material has strong structural nonlinearity.

We conclude that the composite materials have a load decentralization function and a shock-absorbing function by nonlinearity in the elastic region, because rubbers used as a seismic isolation material also absorb shock energy by the nonlinearity in the elastic region.

From the above, it would seem that the material characteristics of the composite materials depend on the direction of constraint displacements. We infer as follows. The deformations are simple compression and tension in the case of $90 \mathrm{deg}$. However, in the case of $45 \mathrm{deg}$., the square lattice imitates the hexagonal honeycomb lattice, as shown Fig. 9. Therefore, the proposed composite materials have a load decentralization function.

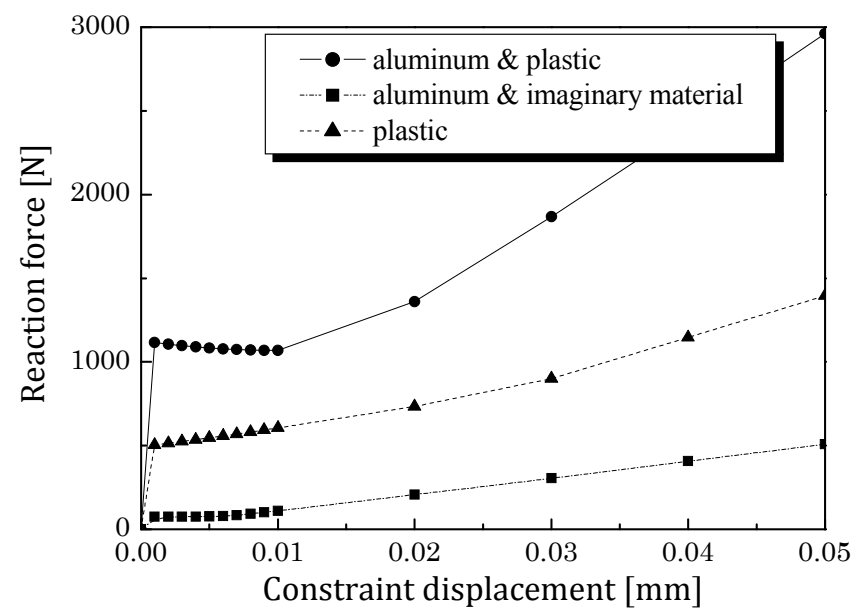

Figure 8: $\quad$ Load-displacement curves (45 deg.).

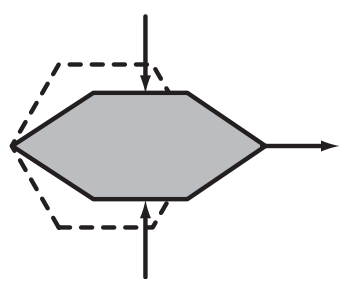

(a) Hexagonal honeycomb lattice

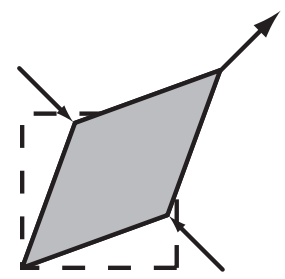

(b) Square lattice

Figure 9: Lattice deformation. 


\subsection{Consideration of a load decentralization function of the composite materials}

Fig.10 shows the relations between the constraint displacements and the reaction forces on the each restraint face. Until the constraint displacement becomes $0.08 \mathrm{~mm}$, the pin on the tension side (POINT 1) receives the greatest load. A reaction force on the compression side (POINT 4) decreases until the constraint displacement becomes $0.01 \mathrm{~mm}$. The reason why the reaction force decreases is that the load is distributed to the $135 \mathrm{deg}$. and $-45 \mathrm{deg}$. directions when a cell between POINT 5 and POINT 4 is deformed. Therefore, we infer that the deformation of cells is a factor to strengthen the nonlinearity of the composite materials.

\section{Conclusion}

We proposed wooden building joints using biomimetic composite materials and discussed the material design. The following has been understood:

1) The proposed composite materials, which consist of a square lattice structure and a filled material, have nonlinear characteristics at the elastic region in the case of a constraint displacement of $45 \mathrm{deg}$. in any direction.

2) It is expected that the materials have a load decentralization function.

3) It is expected that 3D linear analysis will be used for the material design.

4) Using the composite materials for joints can have significant benefits towards the earthquake-proofing of individual houses.

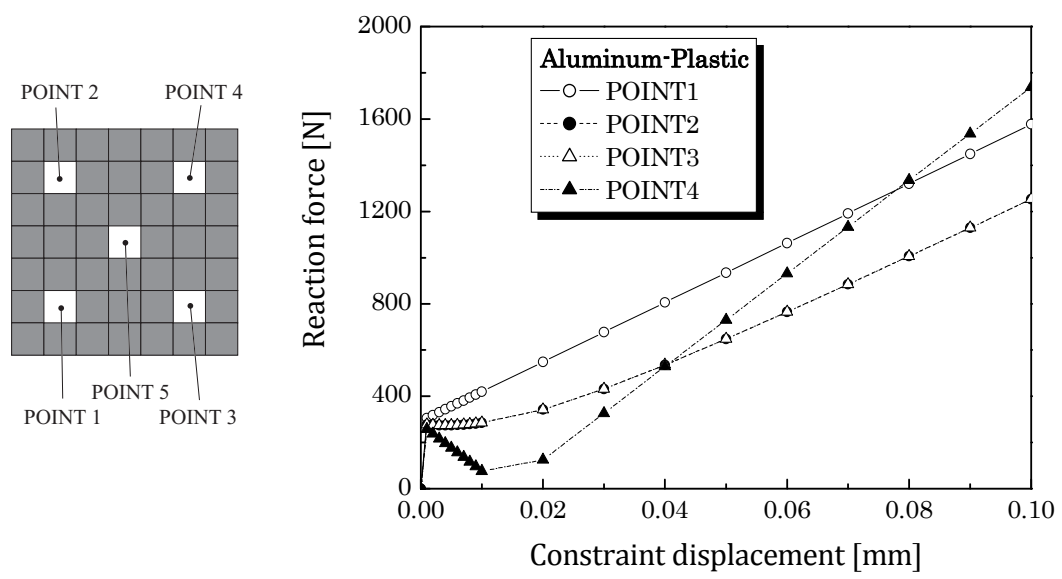

Figure 10: Load-displacement curves (each point). 


\section{References}

[1] M. Inayama, Theoretical Study of Wooden Depressive Behavior, Summaries of technical papers of Annual Meeting Architectural Institute of Japan, pp.13-14, 1989.

[2] M. Inayama et al., Study on compression perpendicular to the grain in wood, Summaries of technical papers of Annual Meeting Architectural Institute of Japan, pp.907-908, 1993.

[3] K. Tanaka et al., Development of solid-air composites by biomimetics superior in load dispersion ability under dynamic loading condition, JSME annual meeting, pp.461-462, 2006. 